\title{
Purification of plasmid DNA vectors by aqueous two-phase extraction and hydrophobic interaction chromatography
}

\author{
Inês P. Trindade ${ }^{\mathrm{a}}$, Maria M. Diogo ${ }^{\mathrm{b}}$, Duarte M.F. Prazeres ${ }^{\mathrm{b}}$ and João C. Marcos ${ }^{\mathrm{a}, *}$ \\ a Centro de Química- IBQF (Pólo de Braga), Universidade do Minho \\ Campus de Gualtar, 4700-320 Braga, Portugal \\ b Centro de Engenharia Biológica e Química, Instituto Superior Técnico \\ 1049-001 Lisboa, Portugal \\ *corresponding author \\ jemarcos@quimica.uminho.pt \\ Phone $+351-253604386$ \\ Fax $+351-253678983$
}

Keywords: Aqueous two-phase systems; liquid-liquid extraction; plasmid; DNA; purification; gene therapy; polyethylene glycol; ammonium sulphate 


\begin{abstract}
The current study explores the possibility of using a polyethyleneglycol(PEG)/ammonium sulphate aqueous two-phase system (ATPS) as an early step in a process for the purification of a model $6.1 \mathrm{kbp}$ plasmid DNA (pDNA) vector. Neutralised alkaline lysates were fed directly to ATPS. Conditions were selected to direct pDNA towards the salt-rich bottom phase, so that this stream could be subsequently processed by hydrophobic interaction chromatography (HIC). Screening of the best conditions for ATPS extraction was performed using three PEG molecular weights $(300,400,600)$ and varying the tie-line length, phase volume ratio and lysate load. For a $20 \%(\mathrm{w} / \mathrm{w})$ lysate load, the best results were obtained with PEG 600 using the shortest tie-line $(38.16 \% \mathrm{w} / \mathrm{w})$. By further manipulating the system composition along this tie-line in order to obtain a top/bottom phase volume ratio of $9.3(35 \% \mathrm{w} / \mathrm{w}$ PEG $\left.600,6 \% \mathrm{w} / \mathrm{w} \mathrm{NH}_{4}\right)_{2} \mathrm{SO}_{4}$ ), it was possible to recover $100 \%$ of pDNA in the bottom phase with a 3 fold increase in concentration. Further increase in the lysate load up to $40 \%(\mathrm{w} / \mathrm{w})$ with this system resulted in a 8-fold increase in pDNA concentration, but with a yield loss of $15 \%$. The ATPS extraction was integrated with HIC and the overall process compared with a previously defined process that uses sequential precipitations with isopropanol and ammonium sulphate prior to HIC. Although the final yield is lower in the ATPS-based process the purity grade of the final pDNA product is higher. This shows that it is possible to substitute the time-consuming two-step precipitation procedure by a simple ATPS extraction.
\end{abstract}




\section{Introduction}

The development of molecular therapies such as non-viral gene therapy and DNA vaccination have increased the need for high quantities of highly purified plasmid DNA (pDNA) [1,2]. One of the bottlenecks of pDNA manufacturing lies in the purification. Although standard molecular biology protocols are available [3], these are not suitable at large-scale [4]. In addition they frequently use toxic reagents that should not be used in the purification of therapeutic products. These constraints have led to an increase in research directed towards the development of alternative methods for the downstream processing of pDNA .

Aqueous two-phase systems (ATPS) constitute an interesting alternative since several features of early processing steps can be combined in only one operation and phase environment is non-toxic for biomolecules. A number of recent references describe the use of ATPS for the extraction of pDNA from cell lysates [5-8]. A thermoseparating ATPS made of (50\% ethylene oxide-50\% propylene oxide)/dextran has been developed for the purification of a $6.1 \mathrm{kbp}$ pDNA from a desalted alkaline lysate [8]. The promising results obtained in this study (100\% pDNA recovery with 80 and $58 \%$ RNA and protein removal respectively) prompted the authors to develop an integrated purification process which combined the thermoseparating ATPS with membrane filtration and chromatography [7]. The more conventional polyethylene glycol (PEG)/salt $\left(\mathrm{K}_{2} \mathrm{HPO}_{4}\right)[5,6]$ ATPS was used to study the partial purification of an $8.5 \mathrm{kbp}$ pDNA vector from a neutralised alkaline lysate [5]. Results showed that by varying PEG molecular weight, pDNA could be directed towards the top $\left(M_{W}<400\right)$ or bottom phase $\left(M_{W}>400\right)$

A typical process for the production of pDNA includes cultivation of an Escherichia coli host, followed by alkaline lysis and a number of purification steps [4]. Since the complexity of alkaline lysates can severely compromise fixed-bed chromatographic operations, pre-purification steps are usually 
necessary. For instances, in a process (Figure 1) based on hydrophobic interaction chromatography (HIC) which has been described for the large scale purification of a pDNA [9], three steps are needed after alkaline lysis and before feeding the HIC column: i) precipitation of pDNA with isopropanol, ii) re-dissolution of pDNA precipitate in an appropriate buffer and iii) precipitation of proteins, RNA and endotoxins with ammonium sulphate $\left(\left(\mathrm{NH}_{4}\right)_{2} \mathrm{SO}_{4}\right)$. This last step further acts as a suitable conditioning step since the salt type $\left(\left(\mathrm{NH}_{4}\right)_{2} \mathrm{SO}_{4}\right)$ and concentration $(2-2.5 \mathrm{M})$ in the final pDNA-containing supernatant match the optimal requirements for a HIC feed. In the current study we explore the possibility of using a PEG/salt ATPS to replace the three early processing steps (Figure 1). In this PEG/salt system, $\left(\mathrm{NH}_{4}\right)_{2} \mathrm{SO}_{4}$ is used instead of the more classical $\mathrm{K}_{2} \mathrm{HPO}_{4}$. Thus, if conditions are selected such that pDNA is directed towards the salt-rich bottom phase, this stream can be injected directly in the HIC column. A $6.1 \mathrm{kbp}$ pDNA was used as a model molecule and screening of the best conditions for ATPS extraction was performed using three PEG molecular weights $(300,400,600)$ and varying the tie-line length, phase volume ratio and lysate load. An adequate system and extraction conditions were then selected and tested in order to assess its feasibility as a replacement of the two pre-purification steps. Further purification by HIC was tested in order to check the compatibility of the ATPS bottom phase with the chromatographic operation. Yield and purity in terms of contaminants RNA, protein and endotoxin were evaluated in the final preparation. 


\section{Experimental}

\section{Chemicals}

PEG 300, 400 and 600 were obtained from Sigma (St. Louis, MO, USA). Ammonium sulphate and potassium acetate were from Merck (Darmstadt, Germany). All the other reagents used were of analytical grade. The 6050 bp (base pairs) ColE1-type plasmid pVAX1/lacZ, designed by Invitrogen (Carlsbad, CA, USA) for the development of DNA vaccines, was used as a model plasmid. This vector contains the human cytomegalovirus (CMV) immediate-early promoter, the BGH polyadenylation sequence, a kanamycin resistance gene, a pMB1 origin (pUC-derived), a multiple cloning site, a T7 promoter/priming site and a reporter ( $\beta$-galactosidase) gene. Escherichia coli DH5 $\alpha$ from Invitrogen was used as the host strain.

\subsection{Plasmid production}

Escherichia coli cells harbouring plasmid pVAX1/lacZ were cultivated overnight $\left(\mathrm{A}_{600} \approx 3.0\right)$ in 1000 $\mathrm{ml}$ shake flasks containing $250 \mathrm{ml}$ of Luria Bertani medium supplemented with $30 \mu \mathrm{g} / \mathrm{ml}$ of kanamycin (Sigma, St. Louis, MO), at $37^{\circ} \mathrm{C}$ and $180 \mathrm{rpm}$. Growth was suspended at late log phase $(\approx 1.5 \mathrm{~g} / \mathrm{L}$ dry cell weight). Plasmid was then isolated from cells as previously described in reference [5] The final plasmid-containing lysate $(\approx 34 \mathrm{ml})$, obtained from $0.38 \mathrm{~g}$ of cells, was stored at $-20^{\circ} \mathrm{C}$ until further processing with ATPS as described below (sub-section 2.4).

\subsection{Characterization of aqueous two-phase systems}

Binodal curves were determined by titration according to Albertsson [10]. Small amounts of water were added to several biphasic systems of defined composition until a one phase system was obtained. The final composition of the system was then calculated and taken as a binodal point. Tie-lines were 
defined by determining the composition of ammonium sulphate and PEG in the top and bottom phases of systems with a defined total composition. Ammonium sulphate concentration was determined by measuring the conductivity of each phase at $25^{\circ} \mathrm{C}$ after adequate dilution. The correspondent salt concentration was then determined from a calibration curve constructed with salt standards of known concentrations. PEG concentration was determined by refractometry after correcting for the contribution of ammonium sulphate. Tie lines lengths (TLL) were calculated according to the following formula:

$$
\operatorname{TLL}(\% \mathrm{~W} / \mathrm{w})=\sqrt{\Delta \mathrm{P}^{2}+\Delta \mathrm{C}^{2}}
$$

where $\Delta \mathrm{P}$ is the difference between $\mathrm{PEG}$ concentration on the two phases and $\Delta \mathrm{C}$ is the difference between ammonium sulphate concentration of the two phases.

\subsection{Aqueous two-phase extraction}

ATPS were prepared in $15 \mathrm{ml}$ graduated tubes with conical tips by mixing adequate amounts of water, ammonium-sulphate, E. coli lysate and PEG, up to a total weight of $5 \mathrm{~g}$. The components were mixed by tube inversion and the two phases separated by centrifugation at $3000 \mathrm{~g}$ for $10 \mathrm{~min}$. The larger volumes needed for further processing by preparative hydrophobic interaction chromatography were prepared in a similar way by mixing a total amount of $20 \mathrm{~g}$ of components in $50 \mathrm{ml}$ graduated tubes.

\subsection{Precipitation with isopropanol and ammonium sulphate}

For comparative purposes, the pDNA-containing lysates were subjected to sequential precipitations with isopropanol and ammonium sulphate, instead of being processed by aqueous two-phase extraction. According to a previously established methodology [9], the pDNA present in $8 \mathrm{ml}$ of neutralised lysate was precipitated by adding 0.7 volumes of isopropanol $\left(90 \mathrm{~min}\right.$ at $\left.4^{\circ} \mathrm{C}\right)$. Plasmid containing pellets 
obtained by centrifugation at $10000 \mathrm{~g}\left(20 \mathrm{~min}\right.$ at $\left.4^{\circ} \mathrm{C}\right)$ were re-dissolved in $3.5 \mathrm{ml}$ of $10 \mathrm{mM}$ Tris $-\mathrm{HCl}$ buffer (pH 8.0). Next, solid ammonium sulphate was dissolved in the pDNA solution up to a concentration of $2.5 \mathrm{M}$. After $15 \mathrm{~min}$ of incubation on ice, precipitated proteins and RNA were removed by centrifugation at $10000 \mathrm{~g}$ (for $20 \mathrm{~min}$. at $4^{\circ} \mathrm{C}$ ). The pDNA-containing supernatant was then loaded directly onto the preparative HIC column.

\subsection{Hydrophobic interaction chromatography $(\mathrm{HIC})$}

Plasmid DNA in the solutions obtained after aqueous two-phase extraction or after sequential precipitation with isopropanol and ammonium sulphate was further purified by preparative HIC as described previously [9], except that Phenyl Sepharose 6 Fast Flow was used instead of 1,4butanedioldiglycidylether Sepharose 6FF. This replacement of matrix did not cause any modification on the performance of the separation. A XK 16/20 column (Amersham Biosciences, Uppsala, Sweden) was packed with Phenyl Sepharose 6 Fast Flow gel (Amersham Biosciences) up to a $14 \mathrm{~cm}$ height. The column was connected to a FPLC system (Amersham Biosciences) and equilibrated with $1.5 \mathrm{M}$ ammonium sulphate in $10 \mathrm{mM}$ Tris- $\mathrm{HCl}(\mathrm{pH} 8.0)$ at a flow rate of $1 \mathrm{ml} / \mathrm{min}$. Plasmid samples $(500 \mu \mathrm{l})$ were then injected at the same flow rate. Isocratic elution was carried out with $1.5 \mathrm{M}$ ammonium sulphate in $10 \mathrm{mM}$ Tris- $\mathrm{HCl}(\mathrm{pH} \mathrm{8.0)}$, and the absorbance was continuously measured at $260 \mathrm{~nm}$. After elution of unbound species in the flowthrough peak (pDNA) at $1.5 \mathrm{M}$ salt, the ionic strength of the buffer was decreased (Tris- $\mathrm{HCl} 10 \mathrm{mM}, \mathrm{pH}$ 8.0) in a step mode in order to elute bound species. The pDNA-containing fractions were collected and analysed for contaminants and plasmid.

\subsection{Agarose gel electrophoresis}

Samples from top and bottom phases were analysed by horizontal electrophoresis in $1 \%$ agarose gels in TAE buffer (40 mM Tris base, $20 \mathrm{mM}$ acetic acid and $1 \mathrm{mM}$ EDTA, pH 8.0) in the presence of 0.5 
$\mu \mathrm{g} / \mathrm{ml}$ ethidium bromide. The Hyperladder I molecular weight marker used was from Bioline (Randolph, MA, USA). The gels were run at $60 \mathrm{~V}$ for $75 \mathrm{~min}$ and then analysed and photographed using the gel documentation software EagleSight 3.2 from Stratagene (La Jolla, CA, USA).

\subsection{Plasmid quantification}

Plasmid in the two phases was quantified by HPLC using a hydrophobic interaction chromatography analytical column according to the method developed by Diogo et al. 2003 [11]. Briefly, a $4.6 \mathrm{~mm} \times 10$ cm HIC Source 15 PHE PE column from Amersham Biosciences (Uppsala, Sweden) was connected to a HPLC system (Merck Hitachi, Darmstadt, Germany) and equilibrated with $1.5 \mathrm{M}$ ammonium sulphate in Tris- $\mathrm{Cl} 10 \mathrm{mM} \mathrm{pH}$ 8.0. Twenty $\mu \mathrm{l}$ of a sample appropriately diluted in the equilibration buffer were injected and eluted at $1 \mathrm{ml} / \mathrm{min}$. All pDNA isoforms (supercoiled, open circular, linear) eluted at a salt concentration of $1.5 \mathrm{M}$ as a single peak. After that, the salt concentration was kept to 0 $\mathrm{M}$ ammonium sulphate during 0.5 minutes in order to elute the bound species. The column was then again equilibrated to $1.5 \mathrm{M}$ ammonium sulphate during 5.5 minutes. The absorbance was recorded at $260 \mathrm{~nm}$. The plasmid pVAX1-LacZ was quantified through a calibration curve constructed with standards of the model plasmid (5 to $50 \mu \mathrm{g} / \mathrm{ml}$ ).

\subsection{Protein quantification}

Total protein in both phases was quantified using the Bradford method [12]. In order to reduce interference from ATPS components, adequate dilutions of the samples were performed and read against blanks with the same dilution and prepared as follows. First, a mixture of the buffers used in the preparation of the lysates was prepared with exactly the same final composition: $12.5 \mathrm{ml}$ of TE buffer (50 mM glucose, $25 \mathrm{mM}$ Tris- $\mathrm{HCl}, 10 \mathrm{mM}$ ethylenediamine tetra-acetic acid (EDTA), pH 8.0) plus $12.5 \mathrm{ml}$ of a pre-chilled $200 \mathrm{mM} \mathrm{NaOH}, 1 \%(\mathrm{w} / \mathrm{v})$ sodium dodecyl sulphate (SDS) solution plus $9.4 \mathrm{ml}$ 
of a solution of $3 \mathrm{M}$ potassium acetate, $11.5 \%(\mathrm{v} / \mathrm{v})$ glacial acetic acid. Then, bottom and top blank samples were obtained by preparing aqueous two-phase systems with the same PEG/salt composition but replacing the $E$. coli alkaline lysate with the three-buffer mixture. Concentrations were determined from a calibration curve using Bovine Serum Albumin (BSA, Sigma) as standard in distilled water. Previously it was confirmed that similar calibrations curves are obtained using either the diluted phases or distilled water as solvent (unpublished results).

\subsection{Endotoxin analysis}

Endotoxin contamination was assessed by using the kinetic-QCL Limulus amoebocyte lysate (LAL) assay kit from Biowhittaker (Walkersville, MD, USA) according to the manufacturer instructions. The detection level for the method used here was $0.005 \mathrm{EU} / \mathrm{mL}$. 


\section{Results and Discussion}

\subsection{Effect of polymer molecular weight}

PEG molecular weight of 300, 400 and 600 were used to assess the influence of this factor in partition and purification of the plasmid in the PEG-( $\left(\mathrm{NH}_{4}\right)_{2} \mathrm{SO}_{4}$ systems. These systems were prepared with a composition closer to the binodal as possible and with a phase volume ratio of approximately 1.0 . The lysate load was $20 \%(\mathrm{w} / \mathrm{w})$. Agarose gel electrophoresis (results not shown) indicates that pDNA and RNA accumulate in the top phase of systems prepared with PEG 300 and PEG 400. However in systems prepared with PEG 600 pDNA accumulates in the bottom phase whereas RNA remained in the top phase. These results were confirmed by quantification of pDNA in both phases (Table 1). The fact that pDNA yields lower than $100 \%$ were obtained in the three systems studied indicates some loss to the interphase. This partitioning behaviour of pDNA is similar to what had been previously found in PEG-K ${ }_{2} \mathrm{HPO}_{4}$ systems with 2.7, 7.1 and $8.5 \mathrm{kbp}$ plasmids [5]. Although the plasmid size and salt composition are different, the factors governing partition are apparently the same. It can be concluded that at least in PEG-( $\left(\mathrm{NH}_{4}\right)_{2} \mathrm{SO}_{4}$ and PEG- $\mathrm{K}_{2} \mathrm{HPO}_{4}$ systems, plasmid size does not influence partition, as opposed to what happens with proteins. On the other hand the salting out effect of the two salts $\left(\left(\mathrm{NH}_{4}\right)_{2} \mathrm{SO}_{4}\right.$ and $\left.\mathrm{K}_{2} \mathrm{HPO}_{4}\right)$ is very similar. In PEG-salt systems the partition of biological macromolecules is determined mainly by two factors: the salting-out ability of the salt phase and the exclusion limit of the polymer phase. Briefly, if the type and concentration of salt in the bottom phase is appropriate to diminish the interactions between water and the macromolecule, this will be directed to the upper phase as long as there is sufficient space to accommodate the solute. In fact it is well known that in solutions of large linear polymers a net is formed limiting the molecular weight of the molecules that could accommodate on it. The space available for other molecules is known as excluded volume and decreases with the increase in molecular weight of the polymer. According to the 
Hofmeister series the salting-out ability of $\mathrm{PO}_{4}^{3-}$ is higher than $\mathrm{SO}_{4}{ }^{2-}$ but that of $\mathrm{K}^{+}$is lower than of $\mathrm{NH}_{4}{ }^{+}$. However in the $\mathrm{K}_{2} \mathrm{HPO}_{4}$ solution the fraction of $\mathrm{PO}_{4}{ }^{3-}$ should be low and $\mathrm{HPO}_{4}{ }^{2-}$ should have less salting-out ability than the former ion. This should give an overall similar salting out effect.

Protein quantification in each phase further showed that proteins, like RNA, partition exclusively to the top phase (Table 1). This behaviour has been previously observed with low molecular weights PEG/salt systems [13]. According to the preliminary results reported in this sub-section, PEG 600 systems were selected for further studies.

\subsection{Effect of tie-line length}

The effect of tie-line length on the partition and purification of pDNA was studied by using a PEG 600 ATPS with a $20 \%(\mathrm{v} / \mathrm{v})$ lysate load. Systems with four distinct compositions were selected and the tieline lengths were calculated by determining the composition of the upper and bottom phase (Fig 2). After pDNA partitioning, quantitative results were obtained by analysing both phases (Table 2). As before, protein accumulated in the top phase for all tie-line lengths, while pDNA accumulated in the bottom phase. However, the yield decreased with the increase in tie-line length. For the higher tie-line length, no pDNA was detected in either phase. An increase in the tie-line length corresponds to an increase in the concentration of salt on the bottom phase. Due to this higher salting-out ability of the bottom phase, pDNA will be forced to move to the upper phase. However due to the excluded volume effect of PEG 600, accumulation in the top phase is prevented and pDNA trapped in the interphase as a precipitate. Visual inspection confirms an increase in the precipitated material at the interphase and supports this hypothesis. This could also be a reasonable explanation for the relatively low yields previously obtained in the PEG (20\%)- $\mathrm{K}_{2} \mathrm{HPO}_{4}(20 \%)$ systems reported by Ribeiro et al. [5]. Although there is no available data in the literature for the tie-line length of the system used then, the highest tie- 
line length reported (41.5\% w/w, for a composition of PEG (18.3\%) - $\mathrm{K}_{2} \mathrm{HPO}_{4}(17.4 \%)$ [13]) points to a tie-line length of around $50 \% \mathrm{w} / \mathrm{w}$, thus supporting the substantial reduction of the plasmid yield. Accordingly to this results a tie-line length of 38.16 was selected for further studies.

\subsection{Effect of phase volume ratio}

The first steps of any purification process should combine high yields with a concentration of the target molecule. In ATPS this could be easily achieved by reducing de volume of the phase where the target compound is collected relatively to the feed volume. In the present case this means increasing the top/bottom phase volume ratio. This procedure will only work as long as the limit of solubility of the target compound is not attained and exceed in the accommodating phase. To test the feasibility of this approach in the current process, several PEG 600 systems with increasing phase volume ratio and with compositions along the optimal (shortest) tie-line $(38.16 \%(\mathrm{w} / \mathrm{w})$ length) were prepared. The partition and purification results were evaluated for a $20 \%(\mathrm{w} / \mathrm{w})$ lysate load (Table 3). The concentration of pDNA in the bottom phase increased with the phase volume ratio. This means that the pDNA does not truly partitions between the phases. Rather, pDNA behaves as being insoluble in the top phase, and accumulates in the bottom phase as long as its solubility limit is not exceeded. Thus, its concentration in the bottom phase increases with an increase in the phase volume ratio. Although some protein also appears in the bottom phase for the highest phase volume ratio, contamination does not exceed $0.08 \mu \mathrm{g}$ protein per $\mu \mathrm{g}$ of pDNA. Given the high yields obtained and the almost 3 -fold concentration of the pDNA relatively to the initial lysate, further studies were conducted with a PEG 600 and $\left(\mathrm{NH}_{4}\right)_{2} \mathrm{SO}_{4}$ composition of 35 and $6 \%$ w/w respectively, which corresponds to a tie line length of $38.16 \%$ (w/w) and a phase volume ratio of $9.3(\mathrm{v} / \mathrm{v})$.

\subsection{Effect of lysate load}


In the experiments described in the previous sections, a lysate load of $20 \%(\mathrm{w} / \mathrm{w})$ was used. An increase on this lysate load would be advantageous since larger feed volumes could be processed by the ATPS. However the concomitant increase in the amounts of both pDNA and contaminants in the systems could result in a decrease of performance of the ATPS, if limits of solubility are exceeded. A set of experiments was then carried out by varying the lysate load up to $40 \%(\mathrm{w} / \mathrm{w})$. The system selected in the previous section was used for these studies. The results are shown in Table 4. Although the concentration of pDNA on the bottom phases increased with the load almost 8-fold relatively to the concentration in the initial lysate, an increase in protein contamination was also observed. In addition, the pDNA yield decreased and precipitated material could be observed in the bottom phases of systems with 30 and $40 \%(\mathrm{w} / \mathrm{w})$ lysate load. This material is most certainly protein that has exceeded its limit of solubility, but some pDNA could also co-precipitate.

In view of the previous results, the system more appropriate to be used as a preliminary step is the one which uses a lysate load of $20 \%$. Although under these conditions the volume of feed processed is not large, a substantial removal of contaminants is achieved. This can be observed very clearly by inspecting the analytical HPLC chromatograms shown in Figure 3. The neutralised lysate feed (Figure 3a) is characterised by the presence of a large amount of impurities (RNA, host DNA and proteins), which elute after pDNA $(0.76 \mathrm{~min})$ as a series of partially overlapping peaks. The HPLC analysis of a sample from the bottom phase obtained after aqueous two-phase partitioning shows a drastic reduction in the amount of impurities (Figure 3b). This is even more striking, if one realises that the peaks eluting after pDNA peak (0.76 min) in Figure $3 \mathrm{~b}$ ) have also the contribution of components from the bottom phase ATPS, as demonstrated by the control HPLC analysis of this phase prepared without lysate (Figure 3c).

\subsection{Integration of ATPS with HIC}


In order to test the feasibility of substituting the sequential precipitations by an aqueous two-phase extraction, the integration of these two procedures with a final HIC purification was compared (Figure 1). For the extraction step a lysate load of $20 \% \mathrm{w} / \mathrm{w}$ in a system composed of $34 \% \mathrm{w} / \mathrm{w}$ PEG 600 and $7 \% \mathrm{w} / \mathrm{w}\left(\mathrm{NH}_{4}\right)_{2} \mathrm{SO}_{4}$, (tie-line length $38.16 \% \mathrm{w} / \mathrm{w}$ and phase ratio $6.2 \mathrm{v} / \mathrm{v}$ ) was used. The system with the higher phase volume ratio $(9.3 \mathrm{v} / \mathrm{v})$ was not selected because even for a $20 \mathrm{~g}$ system in a $50 \mathrm{ml}$ tube the volume of bottom phase was too low for HIC processing. Furthermore, higher volumes of ATPS were not suitable for processing at a small laboratory scale. However given the good results also obtained with the ATPS selected for further HIC processing, it is expected that results could provide conclusions regarding the suitability of the ATPS extraction. The same alkaline lysate was processed in parallel by the ATPS-based and precipitation-based processes. Both processes were able to substantially reduce the amount of impurities. The reduction in protein content was $98 \%$ in both cases (Table 5). RNA clearance, as seen on an agarose gel was also similar (Figure 4). The reduction in the endotoxin load was slightly better when using the ATPS (68vs $51 \%$, Table 5). However the pDNA yield $(75.4 \%)$ of the ATPS process was lower than what had been found in the previous experiments. This is probably due to the higher scale used and the different composition of the lysate. The samples obtained by both methods were then processed by HIC. Samples of the pDNA pool collected were then analysed for pDNA and impurities (Table 5 and Figure 4). The two preparative chromatograms were similar, showing as characteristic features a first peak of non-retained pDNA, a second broader peak of weakly retained species, and a third peak obtained after running a reverse salt step gradient (Figure 5). Nevertheless, it should be kept in mind that components of the ATPS are likely present in the second peak of the chromatogram shown in figure 5a), as also obtained by HIC-HPLC analysis (figure 3c).

Although the yield is better in the preliminary steps and chromatographic step with the precipitation method, the final pDNA preparation has a high purity grade when obtained by the ATPS method. However it must be pointed out that the HIC conditions used were not optimized for the ATPS sample - 
this could be a reasonable justification for the low yields obtained. Further optimization of the HIC step together with the utilization of the ATPS optimized conditions should give comparable yields.

Given its simplicity and the good results obtained this seems to be a good alternative to replace the previous used precipitation method.

\section{Conclusions}

This work shows that it is possible to substitute a three-step, time consuming, pre-purification, conditioning and concentration procedure before HIC purification of plasmids by a simple and fast one step ATPS extraction. The PEG 600 - ammonium sulphate ATPS system selected allows the removal of most RNA and protein contaminants together with a substantial removal of endotoxins. However, extractions conditions should be carefully selected so that systems close to the binodal (short tie-line) are used. Higher tie-lines greatly reduce the yield by accumulation of pDNA in the interface. In addition, pDNA could be concentrated almost 3 and 8-fold relatively to lysate loads of 20 and $40 \%$ $(\mathrm{w} / \mathrm{w})$ respectively. In the later case, however, a small decrease in yield is observed together with the precipitation of material in the bottom phase. Finally, a process which integrates ATPS extraction with HIC gives a final pDNA product with a higher purity (but lower yield) when compared with the conventional precipitation-based process.

\section{Acknowledgements}

This work was supported by the Portuguese Ministry of Science and Technology (POCTI/BIO/47245/2002). 


\section{References}

[1] M. Schleef, in A. Mountain, U. Ney, D. Schomburg (Editors), Recombinant Proteins, Monoclonal Antibodies and Therapeutic Genes, Wiley-VCH, Weinheim, 1999, p. 443.

[2] M. Marquet, N.A. Horn, J.A. Meek, BioPharm September (1995) 26.

[3] J. Sambrook, E. Fritsch, T. Maniatis, Molecular Cloning - A Laboratory Manual, Cold Spring Harbor Laboratory Press, New York, 1989.

[4] D.M.F. Prazeres, G.A. Monteiro, G.N.M. Ferreira, M.M. Diogo, S.C. Ribeiro, J.M.S. Cabral, in M.R. El-Gewely (Editor), Biotechnol. Ann. Rev., Elsevier, Netherlands, 2001, p. 1.

[5] S.C. Ribeiro, G.A. Monteiro, J.M.S. Cabral, D.M.F. Prazeres, Biotechnol. Bioeng. 78 (2002) 376.

[6] S. Ribeiro, G. Monteiro, G. Martinho, J. Cabral, D. Prazeres, Biotechnol. Lett. 22 (2000) 1101.

[7] C. Kepka, R. Lemmens, J. Vasi, T. Nyhammar, P.-E. Gustavsson, J. Chromatogr. A 1057 (2004) 115.

[8] C. Kepka, J. Rhodin, R. Lemmens, F. Tjerneld, P.-E. Gustavsson, J. Chromatogr. A 1024 (2003) 95.

[9] M. Diogo, S. Ribeiro, J. Queiroz, G. Monteiro, N. Tordo, P. Perrin, D. Prazeres, J. Gene Med. 3 (2001) 577.

[10] P.A. Albertsson, Partition of Cell Particles and Macromolecules, John Wiley and Sons, New York, 1985.

[11] M.M. Diogo, J.A. Queiroz, D.M.F. Prazeres, J. Chromatogr. A 998 (2003) 109.

[12] M.M. Bradford, Anal. Biochem. 72 (1976) 248.

[13] B.Y. Zaslavsky, Aqueous Two-Phase Partitioning: Physical Chemistry and Bioanalytical Applications, Marcel Dekker, New York, 1995. 